\section{(2) OPEN ACCESS}

\title{
Impact of a community-based cardiovascular disease service intervention in a highly deprived area
}

\author{
Jennifer Downing (D) , ${ }^{1}$ Tanith C Rose (D) , ${ }^{2}$ Pooja Saini, ${ }^{3}$ Bashir Matata, ${ }^{4}$ \\ Zoe McIntosh, ${ }^{5}$ Terence Comerford, ${ }^{6}$ Keith Wilson, ${ }^{4}$ Allan Pemberton, ${ }^{6}$ \\ Lesley M Harper, ${ }^{7}$ Matthew Shaw, ${ }^{4}$ Konstantinos Daras, ${ }^{8}$ Ben Barr (i) ${ }^{2}$
}

\begin{abstract}
- Additional material is published online only. To view please visit the journal online (http://dx.doi.org/10.1136/ heartjnl-2019-315047).

'Department of Molecular and Clinical Pharmacology, University of Liverpool, Liverpool, UK

${ }^{2}$ Department of Public Health and Policy, University of Liverpool, Liverpool, UK ${ }^{3}$ School of Natural Sciences and Psychology, Liverpool John Moores University, Liverpool, UK ${ }^{4}$ Research Department, Liverpool Heart and Chest Hospital NHS Foundation Trust Liverpool, UK

${ }^{5}$ Knowsley Community Services, Liverpool Heart and Chest Hospital NHS Foundation Trust, Liverpool, United Kingdom ${ }^{6}$ NIHR CLAHRC NWC, University of Liverpool, Liverpool, UK ${ }^{7}$ Department of Health Service Research, University of Liverpool, Liverpool, UK ${ }^{8}$ Department of Geography and Planning, University of Liverpool Liverpool, UK
\end{abstract}

Correspondence to Dr Jennifer Downing, Molecular and Clinical Pharmacology, University of Liverpool, Liverpool L69 3GL, UK;

j.downing@liverpool.ac.uk

JD and TCR are joint first authors.

Received 12 March 2019 Revised 25 July 2019 Accepted 31 July 2019 Published Online First 22 August 2019

Check for updates

(C) Author(s) (or their employer(s)) 2020. Re-use permitted under CC BY-NC. No commercial re-use. See rights and permissions. Published by BMJ.

To cite: Downing J, Rose TC, Saini P, et al. Heart 2020;106:374-379.

\section{ABSTRACT}

Objective To examine the effects on emergency hospital admissions, length of stay and emergency re-admissions of providing a consultant-led, communitybased cardiovascular diagnostic, treatment and rehabilitation service, based in a highly deprived area in the North West of England.

Methods A longitudinal matched controlled study using difference-in-differences analysis compared the change in outcomes in the intervention population, to the change in outcomes in a matched comparison population that had not received the intervention, 5 years before and after implementation. The outcomes were emergency hospitalisations, length of inpatient stay and re-admission rates for cardiovascular disease (CVD).

Results Findings show that the intervention was associated with 66 fewer emergency CVD admissions per 100000 population per year ( $95 \% \mathrm{Cl} 22.13$ to 108.98) in the post-intervention period, relative to the control group. No significant measurable effects on length of stay or emergency re-admission rates were observed. Conclusion This consultant-led, community-based cardiovascular diagnostic, treatment and rehabilitation service was associated with a lower rate of emergency hospital admissions in a highly disadvantaged population. Similar approaches could be an effective component of strategies to reduce unplanned hospital admissions.

\section{INTRODUCTION}

Cardiovascular disease (CVD) is the main reported cause of mortality in the UK, responsible for over a quarter of all deaths. ${ }^{1}$ CVD disproportionally affects disadvantaged socioeconomic groups with rates in the most deprived $10 \%$ of the population twice as high as in the least deprived. ${ }^{2}$ Although the incidence of CVD is declining, the burden of the disease on healthcare services is increasing as the population ages. The cost of CVD to National Health Service (NHS) England, currently at $£ 8.96$ billion per year, is therefore estimated to increase annually. ${ }^{2}$ Improving the identification and treatment of CVD, while reducing emergency admissions and length of inpatient stay, has been highlighted as a priority for the NHS in its 5-year Forward View. ${ }^{3}$ The NHS Long Term Plan $^{4}$ also aims to tackle health inequalities between the most and least deprived, and highlights that cause of death from heart disease is the single largest contributor to the life expectancy gap between these groups. There is therefore an urgent need for evidence of effective interventions that improve the management of CVD and reduce unplanned emergency admissions, particularly in disadvantaged populations.

Existing evidence shows that rapid access chest pain clinics provide efficient and effective substitution to cardiology clinic assessment. ${ }^{5}$ Yet, examples indicate that secondary care-based rapid access clinics may be underutilised by older populations and those in poorer socioeconomic circumstances. ${ }^{6}$ This could potentially be due to problems with access, and there is limited published evidence investigating the provision of rapid access clinics in community settings. Community-based heart failure services have been found to improve access and reduce emergency admissions. ${ }^{7}$ Community-based cardiac rehabilitation shows that it is as effective and safe as hospital-based rehabilitation, and rehabilitation within early supported discharge (ESD) stroke services has been associated with reduced length of hospital stay and reduced mortality rates. ${ }^{8}$ While there is some case study evidence for community-based consultant-led diabetes and respiratory services, ${ }^{9}$ there is limited evidence for consultant-led cardiovascular community-based clinics. Although there is evidence for multicomponent approaches to reduce hospital admissions for single conditions such as heart failure ${ }^{10}$ there is a lack of evidence for consultant-led, communitybased integrated cardiovascular services in deprived communities.

To address these gaps, we evaluated a consultant-led, community-based 'one-stop' CVD service implemented in a very deprived community in the North West of England. We examined the impact of providing this service on emergency admissions, length of inpatient stay and emergency re-admissions.

\section{METHODS \\ Setting}

The intervention was implemented between 2010 and 2015 across the district of Knowsley in the North West of England, which is the second most deprived district in England based on the Indices of Multiple Deprivation. ${ }^{11}$ It has the 13th highest CVD mortality rate in those $<75$ years compared with the other 324 districts in England in 2014 $2016^{12}$ and a population of $148560 .^{13}$ 


\section{Study design}

This study was a longitudinal matched controlled study using lower-layer super output areas (LSOA) as the unit of analysis. LSOAs are small geographical areas used by the UK's Office for National Statistics, each typically containing a population of about 1500 people. England is divided into just over 30000 LSOAs. Ninety-eight LSOAs cover the entire population of the intervention area-Knowsley. Each of these intervention LSOAs was matched with 5 control LSOAs located within other districts in the North West region of England, providing 490 matched control LSOAs-that is, 588 LSOAs in total. We used propensity score matching ${ }^{14}$ to ensure that these control areas had similar observed characteristics to the Knowsley LSOAs in the time period before the introduction of the intervention (2005-2009). The matching was based on the gender and age profile of the population, unemployment rate, Indices of Multiple Deprivation, CVD emergency admission rate, prevalence of coronary heart disease (CHD), smoking prevalence, proportion of the population with hypertension with controlled blood pressure, numbers of general practitioners (GPs) per capita serving the population and the distance to the nearest GP practice and hospital (see online supplementary appendix 1 for full details of the matching variables). The nearest neighbour method was used for matching, which selects controls with propensity scores that are closest to that of the intervention LSOAs. ${ }^{15}$ We checked with the regional cardiovascular network that no other similar intervention was implemented in other areas of North West of England and therefore our control populations would not have experienced a similar intervention.

We then compared the change (difference) in outcomes in the intervention population, to the change (difference) in outcomes in a matched comparison population, 5 years before and 5 years after implementation. This difference-in-differences method controls for all time-invariant differences between the intervention and control populations. The key assumption of differencein-differences analysis is the parallel trends assumption. If the trend in the outcome in the intervention and control populations would have been parallel in the absence of the intervention, then the difference between the change in the outcomes between the two groups provides an unbiased estimate of the interventions effect. ${ }^{16}$ We estimated this difference-in-differences parameter using a linear regression model which included interaction terms between intervention group and time period (before and after). This model additionally included a random intercept for each LSOA, and controlled for annual measures of the percent of population aged $50+$ years, percent female, percent unemployed and time trend terms (see online supplementary appendices 2 and 8 for full details of the statistical model, data sources and analysis; see online supplementary appendix 4 for details of the robustness tests).

\section{The service}

Prior to the implementation of the Knowsley CVD service (KCVDS), the Knowsley population was served by a fragmented service delivered by three NHS Trusts which included a CHD team comprising a heart failure nursing service, cardiac rehabilitation and an ESD service. These services were delivered by different organisations until the KCVDS was set up in 2010. KCVDS provided a new integrated 'one-stop' consultant-led service with a co-located nurse-led heart failure clinic to allow access to consultant support, thereby providing integrated cardiovascular care built around the patient, supported by an administration hub. ${ }^{17}$ The service was designed with public engagement to determine the healthcare locations, and key characteristics provided. ${ }^{18}$ The overall service consists of five elements:

- A consultant-led multidisciplinary clinic. Provided from four Primary Care Resource Centres offering same-day assessment and testing in clinic and at home such as echocardiography, electrocardiography, CardioMemo, blood pressure monitoring (24 hours) and lung function test. It also operated as a rapid access chest pain clinic.

- Diagnostics service. In addition to the tests at the consultant-led clinic, direct access to electrocardiography, 24 hours ambulatory blood pressure monitoring and Ankle Brachial Pressure Index was available.

- Community heart failure clinic. Co-located with the consultant-led clinic or provided in patients' homes when needed, in addition, this service provided home tele-monitoring and tele-health services to optimise patients' treatment and training to support patients' understanding of their symptoms and medication.

- Community stroke rehabilitation service. Provides an ESD service for newly diagnosed stroke cases. It consists of a team providing support 7 days a week, to enable weekend discharge.

- Cardiovascular rehabilitation service. Community-based rehabilitation for patients and their carers in the form of education and exercise classes, delivered group or oneto-one (face-to-face or via telehealth care) in three community centre locations or in patient's homes across Knowsley. In 2014, a nurse-led medication titration element was incorporated into the service to enable patients with heart failure or stroke to use the service at an earlier stage, prior to medication optimisation.

The service is accessed by Knowsley residents primarily through GP referral. ${ }^{17}$ The main additions to the pre-existing services were the provision of a consultant-led service at four sites across Knowsley where patients are seen within 10 days of referral and co-locating this service alongside a nurse-led heart failure service to give direct access to a consultant opinion when needed. Furthermore, due to the single provider model, patients can be referred within the service to all required elements without being re-referred by their GP. Other elements of the service such as face-to-face care in patients' homes, telehealth care and community-based rehabilitations services were provided in the pre-existing model of care.

Knowsley GP referral trends to the KCVDS data show a dramatically increasing trend (online supplementary appendix 7), with the highest number of referrals from GPs located in the most deprived LSOAs (online supplementary appendix 7). From 2010-2011 to 2014-2015 (financial year), the clinic has provided care to almost 13000 patients (online supplementary appendix 7). Clinic attendance has been variable, particularly at the outset of the service (with non-attendance as high as 35\%) stabilising to around $10 \%-13 \%$ across the four clinics. Initially, the service was contracted for 3 years, at an estimated total value of $£ 4.5$ million. ${ }^{19}$

\section{Patient and public involvement}

The research question was developed through a collaboration involving local health service providers, public advisors and researchers. Public advisors are members of the public and/or service users who have knowledge of KCVDS and the locality in which it is delivered. The public advisors were involved in a series of meetings agreeing the focus for the research and the planned analysis. Three of the public advisors (TC, KW and AP) 
Table 1 Characteristics of Knowsley and matched control LSOAs in pre-intervention period (2005-2009)

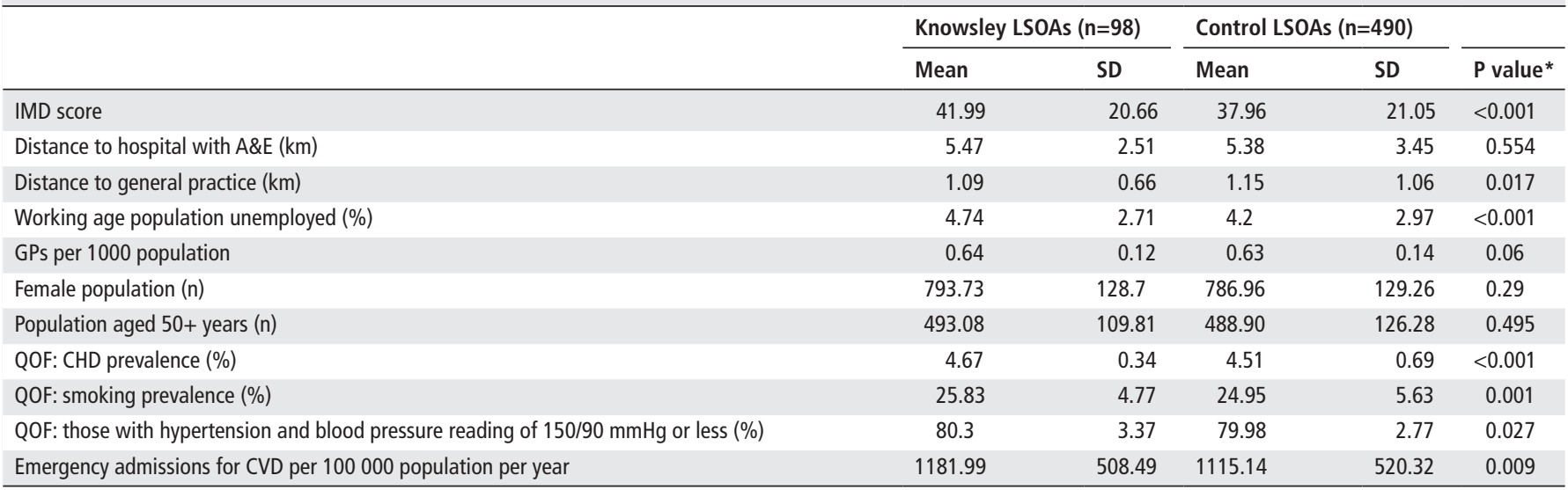

*Statistical significance of the difference between the groups tested using t-tests for normally distributed variables, or the Man-Whitney $U$ test as a nonparametric equivalent. A\&E, Accident and Emergency department; CHD, coronary heart disease; CVD, cardiovascular disease; GP, general practitioner; IMD, Index of Multiple Deprivation; LSOA, lowerlayer super output area; QOF, quality and outcomes framework.

are co-authors of this paper and have contributed to the drafting of the paper and the interpretation of the results.

\section{RESULTS}

Characteristics of the Knowsley and matched control population in the pre-intervention period (2005-2009) are shown in table 1 . Although the control areas at baseline were statistically significantly different from the intervention areas on a number of characteristics, these differences are relatively small, and the difference-in-differences method accounts for these fixed differences in the analysis. All the control areas were also areas with high levels of deprivation, CHD prevalence and CVD admissions (see online supplementary appendix 2 for characteristics of unmatched sample).

Trends in CVD emergency hospital admission rates per year for the Knowsley and control population are shown in figure 1. In the pre-intervention period, emergency admission rates were slightly higher for Knowsley compared with the control population, but the trends were parallel. Following the introduction of the intervention in 2010, admission rates for Knowsley decreased to levels observed in the control population. In more recent years, after the fourth year of the intervention, the admission rates appeared to have started to increase again in Knowsley compared with the control population.

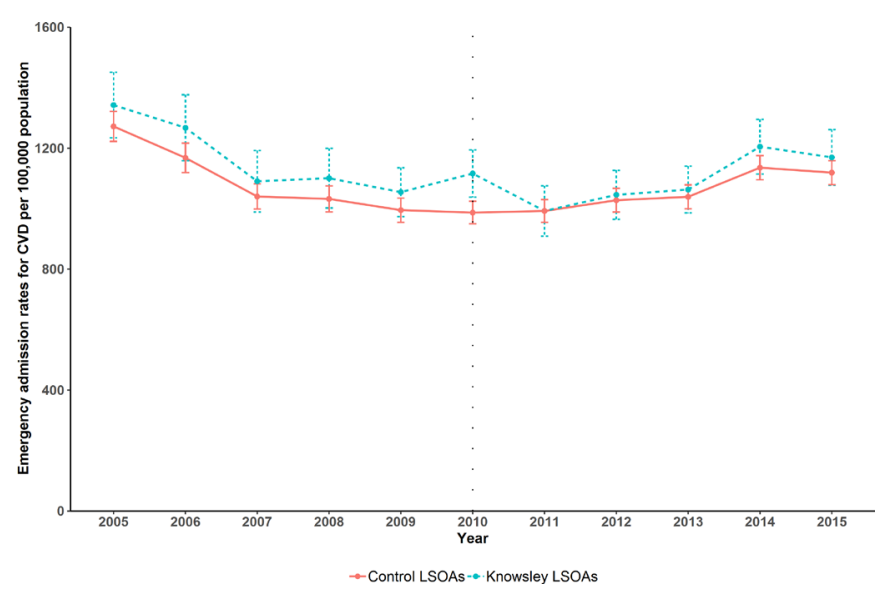

Figure 1 Trends in CVD emergency hospital admission rates per year, by Knowsley and matched control LSOAs, 2005-2015. CVD, cardiovascular disease; LSOAs, lower-layer super output areas.
Results from difference-in-differences analysis for emergency admission rates are shown in table 2. The coefficient for the difference-in-differences estimator indicates that on average the intervention was associated with a lower rate of 66 emergency CVD admissions per 100000 per year (95\% CI 22.13 to 108.98) in Knowsley compared with the control population following the introduction of the intervention. We found that the intervention had no statistically significant effect on reducing length of stay per emergency CVD admission, or emergency re-admission rates (tables 3 and 4).

Analysing the differential effects of the intervention by deprivation and by gender, we found no evidence that these effects differed significantly across these subgroups (see online supplementary appendix 4). The effect on emergency admissions was similar for more deprived areas in Knowsley as for less deprived areas and for both men and women, although there was some evidence to suggest that the intervention was associated with a greater decline in emergency admissions for men compared with women, but this association was not statistically significant at the $5 \%$ level.

\section{Robustness tests}

We found that during the pre-intervention period, there was no significant difference in trends in emergency admission rates between Knowsley and the control population (online supplementary appendix 2), suggesting that the parallel trend assumption was not violated in this analysis. We found no difference in effect when using a Poisson regression model, or when additionally including a random intercept for each matched group (online supplementary appendix 8). We also found that there was no effect when running the analysis using an outcome (emergency admissions for gastrointestinal (GI) infections) that would not plausibly be influenced by the intervention but could have been influenced by unobserved confounding (online supplementary appendix 4). Additionally, using an alternative control group, with controls selected from LSOAs outside the North West region of England, we found similar results (online supplementary appendix 4).

\section{DISCUSSION \\ Principal findings}

We found that an integrated, consultant-led, multicomponent, community-based service was associated with a lower rate of 
Table 2 Result of difference-in-differences analysis showing the change in CVD emergency admissions per 100000 population in Knowsley following the intervention relative to the control group, 2005-2015

\begin{tabular}{|c|c|c|c|c|}
\hline & Coefficient & Lower $95 \% \mathrm{Cl}$ & Upper $95 \% \mathrm{Cl}$ & $P$ value \\
\hline Treatment (Knowsley=1; control=0) & 24.51 & -35.56 & 84.58 & 0.423 \\
\hline Period (post-intervention $=1$; pre-intervention $=0$ ) & -59.92 & -96.19 & -23.64 & 0.001 \\
\hline DiD estimator (treatment* period) & -65.56 & -108.98 & -22.13 & 0.003 \\
\hline
\end{tabular}

Model based on equation shown in online supplementary file and includes random intercept for LSOA, and fixed effects for percent of population aged 50+ years, percent female, percent unemployed and two spline terms for time (full model results are given in online supplementary file).

Model based on 98 Knowsley and 490 control LSOAs, and 6468 observations.

CVD, cardiovascular disease; DiD, difference-in-differences; LSOA, lower-layer super output area.

emergency CVD admissions in a deprived population, and that the intervention had a similar impact across levels of deprivation and between gender groups in this population. Overall, emergency admissions were 66 per 100000 population fewer per year, the equivalent of 97 fewer emergency admissions for the whole Knowsley population per year. Assuming the average reference cost of an emergency admission of $£ 3000$, this would be the equivalent of a cost saving of approximately $£ 300000$ per year, relative to the annual cost of the service of $£ 1.4$ million per year. $^{20}$

Some CVD interventions have been found to be less effective in deprived population. ${ }^{6}$ However, the KCVDS we investigated was at least equal in effectiveness across levels of deprivation and has the potential to reduce health inequalities if it is targeted at more deprived populations. There was, however, some evidence that the effectiveness of the programme began to reduce after 2014 as the number of emergency admissions in Knowsley began to increase at this time. This may be because as the service reached full capacity, it was less able to fully accommodate patient needs, which is substantiated by the service undergoing a staff reorganisation at this time in order to meet demand more effectively. ${ }^{21}$ We found no significant effect on length of hospital stay or on 30-day re-admission rates.

\section{Strengths and limitations}

Our study has a number of strengths. First, we evaluated the KCVDS in its real-life implementation setting, which makes our findings potentially more externally valid than those set in a trial context. Second, the service has been in operation for several years giving a long follow-up period of 5 years. This allowed us to look at whether effects were sustained. Third, we applied a combination of quasi-experimental methods-propensity score matching and difference-in-differences-which provide causal estimates of the intervention if the trends in outcomes would have been parallel in the absence of the intervention. Our approach provides a reasonably large effective sample size of 6468 observations providing reasonable power to identify relatively small effects.
Some limitations, however, remain. We cannot rule out the possibility that different trends in unobserved confounding factors between the two groups may have influenced the results. Although there are clear differences between the intervention and control groups, time-invariant differences between the two groups could not bias the results due to the difference-indifferences methods. ${ }^{22}$ The reasons for matching was to identify groups that were likely to follow similar trend over time, which was confirmed by assessing the parallel nature of the trends in outcomes before the intervention. We additionally controlled for a number of observed confounders. Unobserved confounders therefore could only bias the results if they followed different time trends over time between the intervention and control groups. When repeating the analysis using an outcome that would not plausibly be influenced by the intervention (emergency admissions for GI infections) but could have been influenced by unobserved confounding, such as changes in health service admission thresholds or health provider financial incentives, we found no significant effect of the intervention.

We were able to assess only the impact of the intervention on emergency hospital admissions, and this may not reflect health benefits to the users of these services. Data on other outcomes such as mortality were not available at the geographical level required for this analysis and therefore could not be included. The ecological nature of this study limits the conclusions that can be drawn about individual-level factors, and the results reflect the population-level impact of the KCVDS.

\section{Meaning of the study: possible implications for practice}

Prior to the initiation of KCVDS some services already existed, for example, the ESD service, community rehabilitation and community heart failure services, including the telehealth elements offered. Therefore, the associated benefits of the KCVDS could largely be attributed to a new consultant-led service, and the co-location of this service with the nurse-led heart failure service. This healthcare provision is maximised within a community-based, integrated, single-provider approach

Table 3 Result of difference-in-differences analysis showing the change in length of stay in days per emergency CVD admission in Knowsley following the intervention relative to the control group, 2005-2015

\begin{tabular}{|c|c|c|c|c|}
\hline & Coefficient & Lower $95 \% \mathrm{Cl}$ & Upper $95 \% \mathrm{Cl}$ & $P$ value \\
\hline Treatment (Knowsley $=1$; control $=0$ ) & -2.01 & -3.28 & -0.74 & 0.002 \\
\hline Period (post-intervention $=1$; pre-intervention $=0$ ) & -0.96 & -2.43 & 0.50 & 0.197 \\
\hline DiD estimator (treatment* period) & 1.04 & -0.77 & 2.84 & 0.260 \\
\hline
\end{tabular}

Model includes random intercept for LSOA, and fixed effects for percent of population aged 50+ years, percent female, percent unemployed and two spline terms for time (full model results are given in online supplementary file).

Model based on 97 Knowsley and 489 control LSOAs, and 6446 observations.

CVD, cardiovascular disease; DiD, difference-in-differences; LSOA, lower-layer super output area 
Table 4 Result of difference-in-differences analysis showing the change in CVD emergency re-admissions per 100000 population in Knowsley following the intervention relative to the control group, 2005-2015

\begin{tabular}{|c|c|c|c|c|}
\hline & Coefficient & Lower $95 \% \mathrm{Cl}$ & Upper $95 \% \mathrm{Cl}$ & $P$ value \\
\hline Treatment (Knowsley=1; control=0) & -0.91 & -8.16 & 6.34 & 0.805 \\
\hline Period (post-intervention $=1$; pre-intervention $=0$ ) & -6.98 & -14.01 & 0.05 & 0.052 \\
\hline DiD estimator (treatment* period) & -4.91 & -13.51 & 3.69 & 0.263 \\
\hline
\end{tabular}

Model includes random intercept for LSOA, and fixed effects for percent of population aged 50+ years, percent female, percent unemployed and two spline terms for time (full model results are given in online supplementary file).

Model based on 97 Knowsley and 487 control LSOAs, and 6424 observations.

CVD, cardiovascular disease; DiD, difference-in-differences; LSOA, lower-layer super output area.

that achieves diagnosis and consultant referrals within a 10-day period, thereby meeting key performance indicators.

Access to cardiovascular services has previously been found to be lower among disadvantaged populations potentially widening inequalities. ${ }^{6}$ Whereas some studies have found that community-based, consultant-led clinics for some diseases can be effective at improving access. ${ }^{23}$ However, there has been limited evaluation of CVD services located in disadvantaged communities. Our study indicates that an integrated, consultant-led, multicomponent, community-based CVD service is associated with a lower rate of emergency admissions in a disadvantaged community. There are a number of reasons why this model could improve outcomes. First, access could have been improved as these services were located close to existing community services and public transport routes. ${ }^{18}$ Second, co-locating consultant and nurse-led services could also provide better access to heart failure and cardiology specialist services. This is supported by evidence from the National Heart Failure Audit that found that co-location of services was associated with greater survival rates post hospital discharge in patients with heart failure. ${ }^{24} \mathrm{Co}-\mathrm{lo}-$ cation of services could also improve communication between services and could be a catalysts for improved innovation and quality as has been found elsewhere. ${ }^{25}$ The evidence for recent integration initiatives in the UK has tended to rely on evaluations that have not used quasi-experimental or experimental designs and therefore they provide limited evidence of impact. ${ }^{26}$ Our findings add to the limited evidence-base for the effectiveness of integrated care models for CVD, which aim to coordinate diagnosis, treatment and disease management across the health system so that people receive timely and accessible support.

\section{CONCLUSION}

The KCVDS model represents an example of out-of-hospital care that is associated with a reduction in emergency admissions in highly deprived areas that has the potential to reduce health inequalities. Services such as KCVD may present a feasible approach that can reduce secondary care demand and could provide a model for other disadvantaged areas aiming to reduce emergency admissions.

Acknowledgements The authors thank Liverpool Heart and Chest NHS Foundation Trust, Knowsley Clinical Commissioning Group, Liverpool Clinical Commissioning Group, Knowsley Council and the public advisors who contributed to the study.

Contributors JD and TR are joint first authors. JD and BB conceived the study design. All the team developed the study and contributed to finalising the research question. TR and BB contributed to analysis, KD to indicators and MS to local data provision. BM and ZM supported this work providing information of the nature of the intervention, contextual information and fact-checking the final draft. JD, TR and BB drafted the manuscript and all other authors critically assessed and contributed to the paper and agreed the final manuscript. The public advisers KW, TC and AP contributed throughout the paper and we look forward to continuing our work with them in the future. BB is guarantor for the study. The corresponding author attests that all listed authors meet authorship criteria and that no others meeting the criteria have been omitted.

Funding This study was supported by the National Institute for Health Research Collaboration for Leadership in Applied Health Research and Care North West Coast (NIHR CLAHRC NWC).

Disclaimer The views expressed in this manuscript are those of the authors and do not represent NHS, the NIHR, the Department of Health and Social Care or Liverpool Heart and Chest NHS Foundation Trust. There are no other relationships or activities that could appear to have influenced the submitted work.

Competing interests BM and ZM are employed by Liverpool Heart and Chest NHS Foundation Trust, the provider of KCVDS, and were involved in providing information about the nature of the intervention and providing contextual information upon request when interpreting the results. AP is an elected governor and KW is employed as a patient ambassador at Liverpool Heart and Chest NHS Foundation Trust. Both were involved as public advisors.

Patient consent for publication Not required.

Provenance and peer review Not commissioned; externally peer reviewed.

Data availability statement All data relevant to the study are included in the article or uploaded as supplementary information.

Open access This is an open access article distributed in accordance with the Creative Commons Attribution Non Commercial (CC BY-NC 4.0) license, which permits others to distribute, remix, adapt, build upon this work non-commercially,

\section{Key messages}

What is already known on this subject?

- Cardiovascular disease (CVD) is one of the major causes of mortality in the UK, responsible for over a quarter of all deaths.

- Emergency hospital admission rates are increasing exponentially and are unsustainable.

- There is limited evidence to support the development of outof-hospital care that is effective at reducing CVD emergency admissions, length of stay and emergency re-admissions in disadvantaged populations.

What might this study add?

- This study provides evidence to show that a communitybased, consultant-led CVD service represents a model of out-of-hospital care that is associated with a lower rate of emergency admissions in an area of high deprivation.

- This model of care demonstrates a potential approach for out-of-hospital care that could contribute to a lower rate of emergency admissions.

How might this impact on clinical practice?

- Integrated, community-based, consultant-led, multicomponent CVD services should be implemented to provide better access to services and contribute to a lower rate of emergency hospital admissions, particularly in disadvantaged areas. 
and license their derivative works on different terms, provided the original work is properly cited, appropriate credit is given, any changes made indicated, and the use is non-commercial. See: http://creativecommons.org/licenses/by-nc/4.0/.

ORCID iDs

Jennifer Downing http://orcid.org/0000-0001-7691-1167

Tanith C Rose 0000-0001-5338-0359

Ben Barr http://orcid.org/0000-0002-4208-9475

\section{REFERENCES}

1 Townsend N, Bhatnagar P, Wilkins E, et al. Cardiovascular disease statistics, 2015. London: British Heart Foundation, 2015.

2 Public Health England. Action plan for cardiovascular disease prevention, 2017-2018. London, 2017.

3 NHS England. Next steps of the NHS five year forward view. London: NHS England, 2017.

4 NHS England. The long term plan. England: NHS England, 2019.

5 Sekhri N, Feder GS, Junghans C, et al. Rapid-access chest pain clinics and the traditional cardiology out-patient clinic. QJM 2006;99:135-41.

6 Sekhri $\mathrm{N}$, Timmis A, Hemingway $\mathrm{H}$, et al. Is access to specialist assessment of chest pain equitable by age, gender, ethnicity and socioeconomic status? An enhanced ecological analysis. BMJ Open 2012;2:e001025.

7 Pattenden J, Coulton S, Spilsbury K, et al. The development and impact of the British heart Foundation and the big lottery fund heart failure specialist nurse service in England: final report April 2008. York: The University of York, 2008.

8 Langhorne P, Baylan S. Early supported discharge services for people with acute stroke. Cochrane Database Syst Rev 2017;7:CD000443.

9 Robertson R, Sonola L, Honeyman M, et al. Specialists in out-of-hospital settings: findings from six case studies. London: The King's Fund, 2014.

10 Damery S, Flanagan S, Combes G. Does integrated care reduce hospital activity for patients with chronic diseases? An umbrella review of systematic reviews. BMJ Open 2016;6:e011952.

11 Department for Communities and Local Government. The English indices of deprivation 2015: statistical release, 2015.
12 British Heart Foundation. Cardiovascular Disease Statistics 2018, Chapter 1 Mortality London: British Heart Foundation, 2018. Available: https://www.bhf.org uk/-/media/files/research/heart-statistics/cvd-statistics-2018---chapter-1 [Accessed 19 Mar 2018].

13 Office for National Statistics. Lower layer super output area population estimates (supporting information), 2018. Available: https://www.ons.gov.uk/peoplepopulation andcommunity/populationandmigration/populationestimates/datasets/lowersuperou tputareamidyearpopulationestimates

14 Rosenbaum PR, Rubin DB. The central role of the propensity score in observational studies for causal effects. Biometrika 1983;70:41-55.

15 Austin PC. An introduction to propensity score methods for reducing the effects of confounding in observational studies. Multivariate Behav Res 2011;46:399-424.

16 Dimick JB, Ryan AM. Methods for evaluating changes in health care policy: the difference-in-differences approach. JAMA 2014;312:2401-2.

17 Johnson D. Community cardiovascular service including community rehabilitation for cardiac and stroke patients: service specification. Liverpool, UK: Liverpool Heart and Chest NHS Foundation Trust, 2014.

18 NHS Knowsley Practice Based Commissioners. Clinical service redesign: Knowsley community cardiovascular service. Knowsley Council, 2011.

19 Knowsley Clinical Commissioning Group. Commissioning intelligence report, 2012.

20 Curtis LA, Burns A. Unit costs of health and social care 2018. Kent, UK: University of Kent, 2018.

21 Maclntosh Z, Gossage E, Comerford T, et al. A service evaluation into the effectiveness of Knowsley cardiovascular service. Liverpool, UK: Liverpool Heart and Chest Hospital NHS Foundation Trust, 2018.

22 Angrist JD, Pischke JS. Mostly harmless econometrics: an empiricist's companion. Princeton: Princeton University Press, 2009.

23 Das M, Panter L, Wynn GJ, et al. Primary care atrial fibrillation service: outcomes from consultant-led anticoagulation assessment clinics in the primary care setting in the UK. BMJ Open 2015;5:e009267.

24 Cleland J, Dargie H, Hardman S, et al. National heart failure audit 2011/12. Annual report. NICOR, 2012.

25 Memon AR, Kinder T. Co-location as a catalyst for service innovation: a study of Scottish health and social care. Public Management Review 2017;19:381-405.

26 Ahmad N, Lawrie M, Patel R, et al. Integrated care pilots evaluation: final report. British Heart Foundation, 2015 